\title{
Fluctuations of topological disclination lines in nematics: renormalization of the string model
}

\author{
D. Svenšek* and S. Žumer \\ Department of Physics, University of Ljubljana, \\ Jadranska 19, SI-1000 Ljubljana, Slovenia
}

(November 14, 2018)

\begin{abstract}
The fluctuation eigenmode problem of the nematic topological disclination line with strength $\pm 1 / 2$ is solved for the complete nematic tensor order parameter. The line tension concept of a defect line is assessed, the line tension is properly defined. Exact relaxation rates and thermal amplitudes of the fluctuations are determined. It is shown that within the simple string model of the defect line the amplitude of its thermal fluctuations is significantly underestimated due to the neglect of higher radial modes. The extent of universality of the results concerning other systems possessing line defects is discussed.

PACS number(s): 61.30.Dk, 61.30.Jf, 05.40.-a
\end{abstract}

The fluctuation problem of the nematic disclination line is one of the central yet untackled problems in the field of liquid crystals, despite the fact that topological disclination lines are the most distinct and regularly observed characteristic of the nematic phase [1-4] and are reflected even in its name. Lately they have been playing a role as experimental objects in studies related to cosmology [5-7]. Controlled experiments with isolated lines or pairs of lines can be performed to study their motion [8,9]. Thermal fluctuations of disclination lines can be observed and analyzed simply by polarization microscopy $[10,11]$.

In this paper, we present the solution of the full tensorial fluctuation problem of a straight wedge disclination line with strength $\pm 1 / 2$, in terms of finding the exact eigenmodes. We focus on the soft modes that correspond to string fluctuations (Fig. 1, inset) and challenge the validity of the string model of the disclination line [12]. The main results of our study are not limited to nematics, as our description does not include the physical system-specific elastic anisotropy or hydrodynamic flow. In particular, they do not depend on the winding number of the defect and can be directly applied to disclination lines in smectics- $\mathrm{C}$ and vortex lines in superfluids. The fluctuations of the strength 1 nematic disclination line were studied in the director description [13,14], precluding the string fluctuations. Moreover, the topological $\pm 1 / 2$ disclinations cannot be described by the Frank director elasticity, whereas the integer disclinations are known to be unstable.

The director free energy per unit length — line tension — of a straight disclination line with strength $s$ is

$$
\mathcal{F}_{0}=\pi s^{2} K \ln \frac{R}{r_{0}},
$$

where $K$ is the Frank elastic constant, $R$ the system size and $r_{0}$ a microscopic cutoff. Hence, in a model the disclination line can be considered as a simple string under tension [12, p. 178]. The energy cost of its (overdamped) fluctuation modes (Fig. 1, inset), which can be attributed to the increase in length, $\Delta l=\int d z(\partial u / \partial z)^{2} / 2$, is thus

$$
\Delta F=\frac{1}{2} k^{2} u^{2} \mathcal{F}_{0} \int d z \cos ^{2} k z
$$

where $u$ is the amplitude and $k$ the wave vector of the mode. The string model of a line defect is general [15-17] and not restricted to nematic disclination lines.

Let us set the scene for the complete description of the disclination line in terms of the nematic tensor order parameter Q. Cylindrical coordinates $(r, \phi, z)$ with corresponding $\mathbf{r}$-dependent orthonormal base vectors $\left\{\hat{\mathbf{e}}_{r}, \hat{\mathbf{e}}_{\phi}, \hat{\mathbf{e}}_{z}\right\}$ will be used. The unperturbed disclination line (the ground state, as referred to from now on) coincides with the $z$ axis. The ground state is $z$-independent, hence, the $z$-dependence of the eigenmodes is sinusoidal. In one (elastic) constant approximation, the free energy density $f(Q, \nabla Q)$ reads

$$
f=\frac{1}{2} A \operatorname{Tr} \mathrm{Q}^{2}+\frac{1}{3} B \operatorname{Tr} \mathrm{Q}^{3}+\frac{1}{4} C\left(\operatorname{Tr} \mathrm{Q}^{2}\right)^{2}+\frac{1}{2} L \operatorname{Tr}(\nabla \mathrm{Q} \cdot \nabla \mathrm{Q})
$$

\footnotetext{
*Author for correspondence. Phone: +386 1 4766587, fax: +386 12517281 , e-mail: daniel@fiz.uni-lj.si
} 
and is invariant upon a homogeneous rotation of the Q-tensor. Hence, the Q-eigensystem rotates as $\psi=\psi_{0}+(s-1) \phi$ with respect to the above base vectors when we encircle a defect of strength $s$ located at the origin; $\psi$ is the angle between $\hat{\mathbf{e}}_{r}$ and the tensor axis corresponding to the director in the far-field, while $\psi_{0}$ is the free parameter of the defect configuration, corresponding to the angle between the director axis at $\phi=0$ and the $x$ axis. There is no dependence on $\phi$ other than this rotation, i.e., the scalar invariants of $Q$ (the degree of order and biaxiality) are $\phi$-independent - a "generalized" cylindrical symmetry.

Defining another $\mathbf{r}$-dependent orthonormal triad $\left\{\hat{\mathbf{e}}_{1}, \hat{\mathbf{e}}_{2}, \hat{\mathbf{e}}_{z}\right\}$, where $\hat{\mathbf{e}}_{1}=\hat{\mathbf{e}}_{r} \cos \psi+\hat{\mathbf{e}}_{\phi} \sin \psi$ and $\hat{\mathbf{e}}_{2}=-\hat{\mathbf{e}}_{r} \sin \psi+$ $\hat{\mathbf{e}}_{\phi} \cos \psi$, in the unperturbed configuration the Q-tensor eigensystem coincides with this triad everywhere. Defining further the orthonormal symmetric traceless base tensors [18]: $\mathrm{T}_{0}=\left(3 \hat{\mathbf{e}}_{z} \otimes \hat{\mathbf{e}}_{z}-\mathrm{I}\right) / \sqrt{6}, \mathrm{~T}_{1}=\left(\hat{\mathbf{e}}_{1} \otimes \hat{\mathbf{e}}_{1}-\hat{\mathbf{e}}_{2} \otimes \hat{\mathbf{e}}_{2}\right) / \sqrt{2}$, $\mathrm{T}_{-1}=\left(\hat{\mathbf{e}}_{1} \otimes \hat{\mathbf{e}}_{2}+\hat{\mathbf{e}}_{2} \otimes \hat{\mathbf{e}}_{1}\right) / \sqrt{2}, \mathrm{~T}_{2}=\left(\hat{\mathbf{e}}_{z} \otimes \hat{\mathbf{e}}_{1}+\hat{\mathbf{e}}_{1} \otimes \hat{\mathbf{e}}_{z}\right) / \sqrt{2}, \mathrm{~T}_{-2}=\left(\hat{\mathbf{e}}_{z} \otimes \hat{\mathbf{e}}_{2}+\hat{\mathbf{e}}_{2} \otimes \hat{\mathbf{e}}_{z}\right) / \sqrt{2}$, the ground state Q-tensor components are $\phi$-independent, while the components of an arbitrary perturbation can be translated in $\phi$ not changing the energy. Hence, the $\phi$-dependence of the eigenmode components is sinusoidal.

In the ocean of fluctuations one should first look for the Goldstone modes, as they yield families of slowly relaxing and spatially extensive "soft" fluctuation modes that can be easily observed. "Massive" fluctuations are less interesting, as they are short-living $(\approx 100 \mathrm{~ns})$ and localized $(\approx 10 \mathrm{~nm})$. Besides the homogeneous rotations of the Q-tensor, which in a deformed nematic are soft only in the one constant approximation, in a deformed system there exist additional nontrivial Goldstone modes, in our case the interest is in those corresponding to the displacement of the disclination line. Since translational degrees of freedom are absent in the analysis, the "displacement" is achieved by modifying (perturbing) the order parameter field. Modulating the displacement modes sinusoidally along $z$ results in the string fluctuations of the disclination line (Fig. 1, inset).

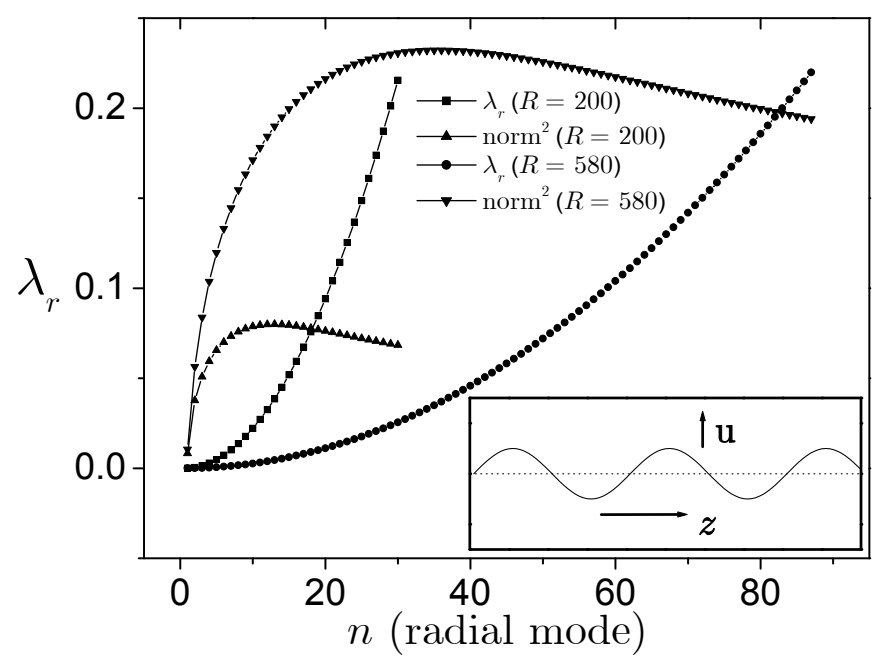

FIG. 1. Radial eigenvalue $\lambda_{r}$ (in units of $1 / \tau$ ) and the norm squared of the radial eigenfunctions (arbitrary units) for two radii of confinement. For $R=200, \lambda_{r}^{(1)}=1.4 \cdot 10^{-5}, \lambda_{r}^{(2)}=4.5 \cdot 10^{-4}, \lambda_{r}^{(3)}=1.4 \cdot 10^{-3}$; note that $\lambda_{r}^{(2)} / \lambda_{r}^{(1)} \approx 30$ ! Inset: the string fluctuation of the disclination line.

Expressing the Q-tensor as $\mathbf{Q}(\mathbf{r}, t)=q_{i}(\mathbf{r}, t) \mathbf{T}_{i}(\mathbf{r})$, the elastic (gradient) part of (3) is

$$
\begin{aligned}
f^{e} & =\frac{L}{2}\left\{\left(\frac{\partial q_{i}}{\partial r}\right)^{2}+\left(\frac{\partial q_{i}}{\partial z}\right)^{2}+\right. \\
& \left.+\frac{1}{r^{2}}\left[\left(\frac{\partial q_{0}}{\partial \phi}\right)^{2}+\left(\frac{\partial q_{1}}{\partial \phi}-2 s q_{-1}\right)^{2}+\left(\frac{\partial q_{-1}}{\partial \phi}+2 s q_{1}\right)^{2}+\left(\frac{\partial q_{2}}{\partial \phi}-s q_{-2}\right)^{2}+\left(\frac{\partial q_{-2}}{\partial \phi}+s q_{2}\right)^{2}\right]\right\}
\end{aligned}
$$

while the homogeneous (gradient-independent) part of (3) is a polynomial in $q_{i}$. We introduce dimensionless quantities $r \leftarrow r / \xi, t \leftarrow t / \tau,(A, B, C) \leftarrow(A, B, C) \xi^{2} / L$, with the correlation length of the degree of order $\xi$, typically a few $\mathrm{nm}$, and the characteristic time $\tau=\mu_{1} \xi^{2} / L$, typically tens of ns, where $\mu_{1}$ is the bare rotational viscosity, i.e., $\gamma_{1}=9 S^{2} \mu_{1} / 2 ; \gamma_{1}$ is the usual rotational viscosity and $S$ the degree of order. Neglecting the hydrodynamic flow, the order parameter dynamics is governed by the time-dependent Ginzburg-Landau equation

$$
\dot{q}_{i}=\nabla \cdot \frac{\partial f}{\partial \nabla q_{i}}-\frac{\partial f}{\partial q_{i}},
$$


where the time derivative vanishes in the ground state.

Owing to the generalized cylindrical symmetry, the ground state involves only $\mathbf{Q}_{0}(\mathbf{r})=a_{0}(r) \mathrm{T}_{0}+a_{1}(r) \mathrm{T}_{1}$, as opposed to perturbations: $\Delta \mathrm{Q}(\mathbf{r}, t)=x_{i}(\mathbf{r}, t) \mathrm{T}_{i}$. The fluctuation eigenmodes $x_{i}$, satisfying $\dot{x}_{i}=-\lambda x_{i}$, are sought by the ansätze

$$
\begin{aligned}
& x_{i}=R_{i, m}(r) \Phi_{i}(m \phi) \sin (k z) \exp (-\lambda t), \quad i=0, \pm 1, \\
& x_{i}=R_{i, n}(r) \Phi_{i}(n \phi) \sin (k z) \exp (-\lambda t), \quad i= \pm 2,
\end{aligned}
$$

where $\Phi_{i}=\cos$ for $i \geq 0$ and $\Phi_{i}=\sin$ for $i<0$ (the global angular phase and the $z$ phase are arbitrary), $m$ is an integer, whereas $n=1 / 2,3 / 2,5 / 2, \ldots$ is a half-integer due to the continuity and differentiability requirements (spinor symmetry of the base tensors)! In the one constant approximation, the sets of components (6) and (7) are not coupled, i.e., in-plane and out-of-plane fluctuations are independent. Furthermore, the $z$-dependence is fully decoupled, i.e., the eigenfunctions $R_{i}(r)$ do not depend on $k$ and the cross-section structure of the disclination line is not affected by the $z$ modulation.

We focus on the in-plane fluctuations. Putting the ansatz (6) into Eq. (5), the eigensystem for the radial functions $R_{i, m}(r)$ remains, with $\lambda=\lambda_{r}+k^{2}$, where $\lambda_{r}$ is the radial eigenvalue:

$$
\begin{aligned}
& \nabla^{2} R_{0, m}+\left(\lambda_{r}-g_{0}(r)-\frac{m^{2}}{r^{2}}\right) R_{0, m}+g_{01}(r) R_{1, m}=0 \\
& \nabla^{2} R_{1, m}+\left(\lambda_{r}-g_{1}(r)-\frac{m^{2}+4 s^{2}}{r^{2}}\right) R_{1, m}-\frac{4 s m}{r^{2}} R_{-1, m}+g_{01}(r) R_{0, m}=0 \\
& \nabla^{2} R_{-1, m}+\left(\lambda_{r}-g_{-1}(r)-\frac{m^{2}+4 s^{2}}{r^{2}}\right) R_{-1, m}-\frac{4 s m}{r^{2}} R_{1, m}=0
\end{aligned}
$$

the $g$ 's are quadratic polynomials of the ground state components. Note that the operator (8)-(10) is self-adjoint. Also note that defects with strengths $s$ and $-s$ are formally equivalent, i.e., changing the sign of the defect and redefining $\mathrm{T}_{\{-1,-2\}} \rightarrow-\mathrm{T}_{\{-1,-2\}}$ conserves the sign of $s$ in the equations.

For an infinite system, one can determine the homogeneous displacement mode directly by construction. Putting $\mathrm{Q}_{0}(\mathbf{r}-\mathbf{u})=\mathrm{Q}_{0}(\mathbf{r})+\Delta \mathrm{Q}(\mathbf{r})$, the perturbation $\Delta \mathrm{Q}$ corresponding to the displacement $\mathbf{u}$ is $\Delta \mathrm{Q}(\mathbf{r})=-\mathbf{u} \cdot \partial \mathrm{Q}_{0} / \partial \mathbf{r}$, which reads

$$
x_{0}(\mathbf{r}) \mathbf{T}_{0}+x_{1}(\mathbf{r}) \mathbf{T}_{1}+x_{-1}(\mathbf{r}) \mathbf{T}_{-1}=-\mathbf{u} \cdot\left(\hat{\mathbf{e}}_{r} \frac{\partial a_{0}}{\partial r} \mathbf{T}_{0}+\hat{\mathbf{e}}_{r} \frac{\partial a_{1}}{\partial r} \mathbf{T}_{1}+\hat{\mathbf{e}}_{\phi} 2 s \frac{a_{1}}{r} \mathbf{T}_{-1}\right) .
$$

Thus, $x_{i}$ in (11) are the eigenfunctions corresponding to $\lambda_{r}=0$ and $m=1$. The lowest family of string modes is generated by adding the $z$-dependence. Hence, $\lambda=k^{2}$, or $\lambda=L k^{2} / \mu_{1}=K k^{2} / \gamma_{1}$ in physical units $\left(K=9 S^{2} L / 2\right)$, which is thus the exact result for the relaxation rate of the string mode, not obtainable in the director picture.

The energy cost of an arbitrary fluctuation eigenmode is given by $\Delta F(\mathbf{x})=\lambda \int d V x_{i}^{2} / 2$. For the homogeneous displacement mode (11), integrating over $\phi$, this results in

$$
\Delta F=\frac{1}{2} k^{2} u^{2} \underbrace{\int r d r\left[\left(\frac{\partial a_{0}}{\partial r}\right)^{2}+\left(\frac{\partial a_{1}}{\partial r}\right)^{2}+4 s^{2}\left(\frac{a_{1}}{r}\right)^{2}\right]} \int d z \cos ^{2} k z .
$$

Comparing (12) (multiplied by $L \xi$ to revert to physical units) with the form of the energy cost (2) of the simple string fluctuation, a line tension can be defined for the disclination line, as indicated by the underbrace. If the string model was accurate, the line tension defined in (12) would be the actual free energy (3) of the unperturbed disclination line per unit length. One verifies that it is exactly the elastic free energy (4) per unit length, whereas the homogeneous contributions are absent. Only the elastic terms contribute to the line tension! This is a general statement - any other homogeneous contributions, should they exist, e.g., electric, do not enter the line tension. It must be stressed that within the isotropic order parameter elasticity this finding is universal. Physically it means that the string fluctuation conserves the volume of the defect line, as opposed to the fluctuation of a real string. Thus, far from the defect core, where the homogeneous free energy contributions vanish, the string model is exact. Moreover, using the bulk values of $a_{0}$ and $a_{1}$, the line tension (1) is recovered exactly. Due to the director distortion, however, this regime is not approached exponentially but by a power-law.

Let us now focus on the $m=1$ fluctuations with $\lambda_{r}>0$. Unlike $R_{-1}$, the functions $R_{0}$ and $R_{1}$ are "localized" (Fig. 2), since $g_{0}, g_{1} \rightarrow$ const. $>0$ (of order 1), while $g_{-1} \rightarrow-4 s^{2} / r^{2}$. Asimptotically, $R_{-1, m}(r)$ behaves as a combination of Bessel functions $J_{m}\left(\sqrt{\lambda_{r}} r\right)$ and $Y_{m}\left(\sqrt{\lambda_{r}} r\right)$, whereas $R_{\{0,1\}}$ first decay as $\exp (-r) / \sqrt{r}$, followed by a 
power-law asymptotics (Fig. 2, inset): $R_{\{0,1\}, m} \propto 4 m s R_{-1, m} / r^{2}$. In solving (8)-(10), we confine the system at a radius $R$ with the restriction $R_{i, m, n}(R)=0$ in order to get a discrete spectrum $\lambda_{r}^{(n)}, n=1,2,3, \ldots$ One might argue that this boundary condition is rather unphysical. In a real sample, however, one never deals with an ideally isolated disclination line - the observed line is surrounded by other defects and irregularities. The boundary condition should therefore be viewed as due to an effective confinement. In the limit $R \rightarrow \infty$, physical observables should be only weakly dependent of $R$ anyhow, e.g., like the free energy (1). Furthermore, if one uses the boundary condition $R_{i}^{\prime}(R)=0$, the lowest eigenmode is of the growing type, $\lambda_{r}<0$, where $\lambda_{r} \propto 1 / R^{2}$, reflecting the instability of the defect towards the escape from the system. This finite-size effect is often manifest in experimental situations.

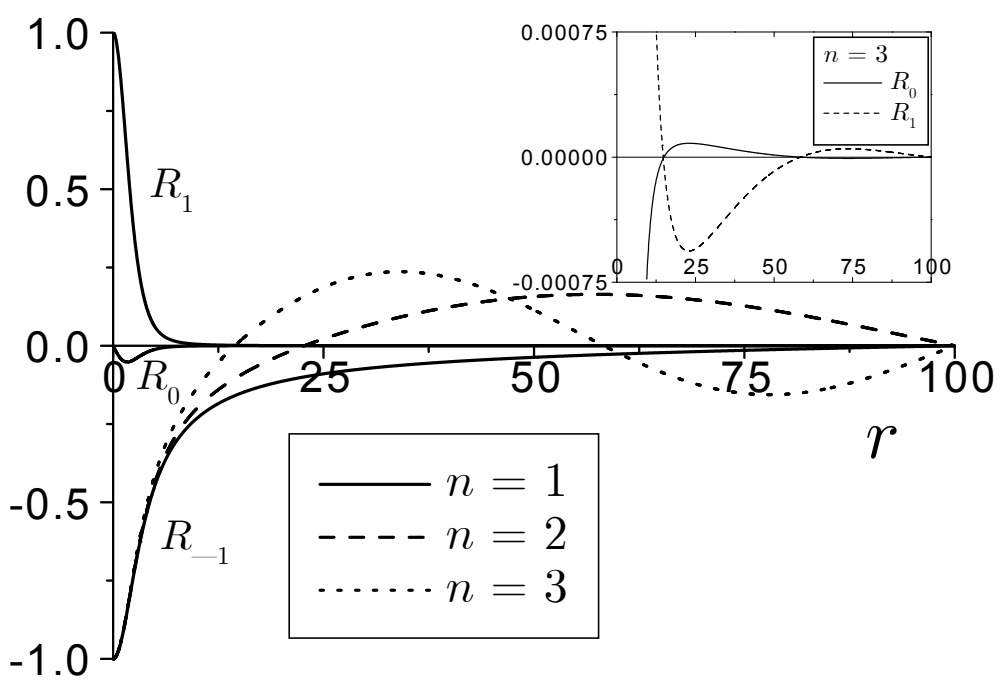

FIG. 2. Three lowest radial eigenfunctions (not normalized) for $m=1$. Functions $R_{0,1, n}$ and $R_{1,1, n}$ overlap on the scale of the big graph; the detail for $n=3$ is shown in the inset - note the power-law tail and the zeros, which coincide with the zeros of $R_{-1}$.

The boundary conditions at $r=0$ are obtained by finding the analytic behavior of the ground state and the perturbations for small $r: a_{0}^{\prime} \rightarrow 0, a_{1} \rightarrow$ const. $\times r^{|2 s|}, R_{0, m} \rightarrow r^{m}$, and $R_{ \pm 1, m} \rightarrow \pm a_{1} r^{|m-2 s|}+a_{2} r^{|m+2 s|}$. The eigensystem (8)-(10) is discretized and efficiently solved by a multidimensional Newton relaxation method [19, p. 588], Fig. 2. In the relevant limit $R \gg \xi$, the radial dependence of the lowest modes is identical for $r \ll R$ and given by (11), except for the normalization, i.e., each of these modes contributes to the displacement $\mathbf{u}$ of the central part! Still in the limit $R \gg \xi$, the difference $\sqrt{\lambda_{r}^{(n+1)}}-\sqrt{\lambda_{r}^{(n)}}$ approaches $\pi / R$ when going to higher modes, i.e., $\lambda_{r}^{(n)} \rightarrow(\text { const. }+n \pi / R)^{2}$, Fig. 1. Similarly, for $n \gg 1$, the eigenvalue of the $n$th mode scales as $\lambda_{r}^{(n)} \propto 1 / R^{2}$ with the system size $R$.

We have shown that within the simple string model the degrees of freedom of the disclination are cut to the lowest $m=1$ mode only. Thus, quite universally, the contributions of the higher modes represent a renormalization of the model. Thermal fluctuation amplitude at $r=0$ of the $k$ th Fourier component ( $z$ direction) is

$$
\left\langle\Delta Q_{i}^{2}(r=0, m=1, k)\right\rangle=2 \sum_{n}\left\langle c_{k, n}^{2}\right\rangle R_{i, 1, n}^{2}(0),
$$

with the radial functions normalized, $\left\langle c_{k, n}^{2}\right\rangle=k_{B} T / L \xi\left(k^{2}+\lambda_{r}^{(n)}\right)$ given by equipartion, and the factor 2 coming from the twofold degeneracy ( $\cos \phi$ and $\sin \phi$ in the angular part). The displacement $\left\langle\mathbf{u}_{k}^{2}\right\rangle$ of the line is obtained from (13) by means of (11). If only the lowest radial mode is taken into account in (13), which is equivalent to using the nonrenormalized string model, the thermal amplitude is obviously underestimated, Fig. 3, or conversely, the extracted value of $K$ is too low. The error increases rather slowly (logarithmically) with $R$; for experimentally relevant scales it is of the order of $100 \%$. The failure of the string model is best demonstrated in the limit $R \rightarrow \infty$ : for a fixed and nonzero $k,\left\langle\mathbf{u}_{k}^{2}\right\rangle \rightarrow 0$ if only the lowest radial mode is taken into account, since the norm (squared) of the mode is logarithmically diverging when $R \rightarrow \infty$. Alternatively, the same can be seen by noting the logarithmic divergence of the line tension (1). 


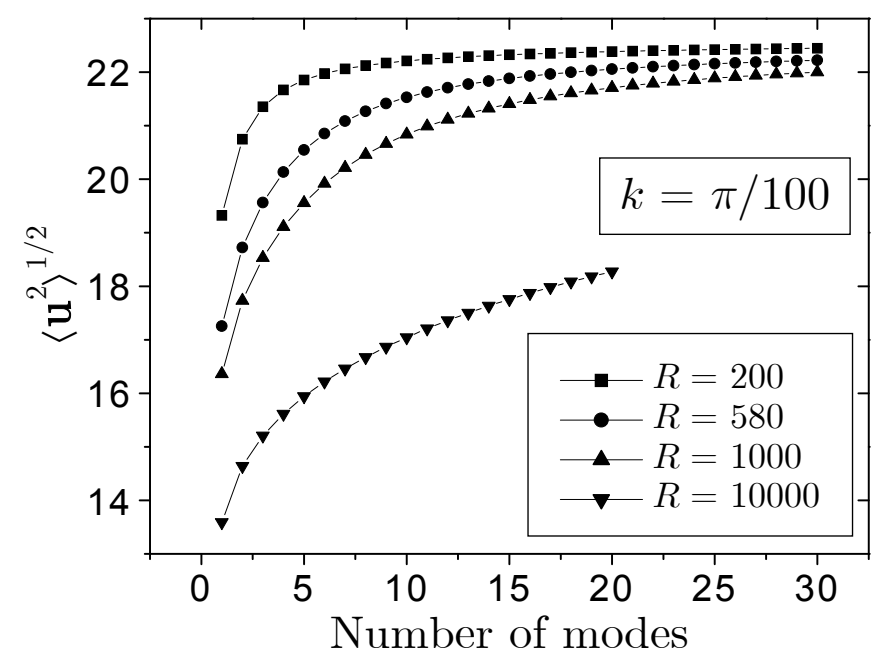

(a) $k=\pi / 100$

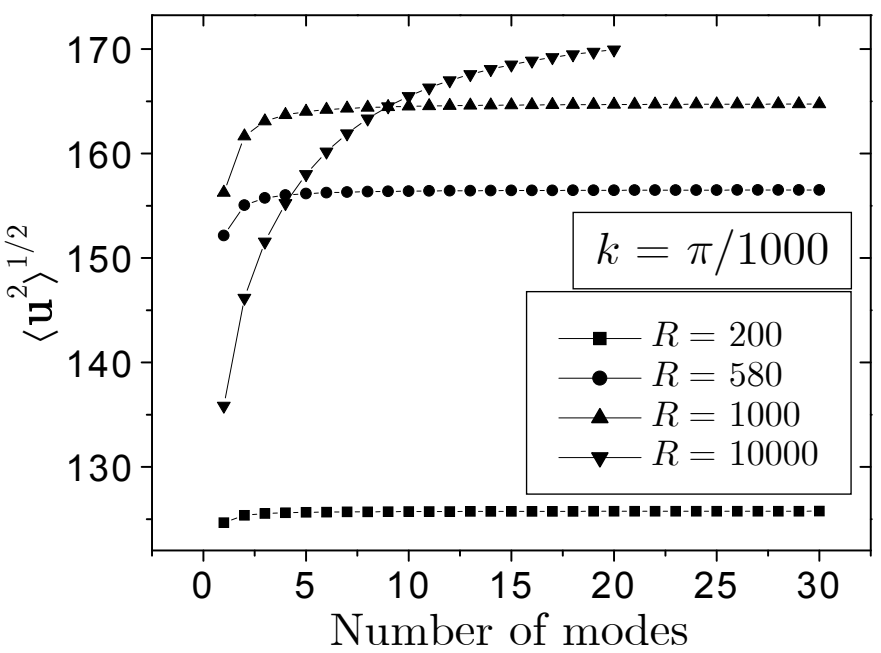

(b) $k=\pi / 1000$

FIG. 3. rms displacement of the disclination line (the length unit is $\xi$ ) vs. the number of radial modes summed in the regimes of a) weak and b) severe confinement; $n=1$ corresponds to the non-renormalized string model. The maximum size of the computational domain is $R=580$, the values beyond this range $(R=1000, R=10000)$ are extrapolated.

Thus, to simulate or analyze a real observation of the disclination line fluctuations, e.g., by polarizing microscopy, for a given $k$ one has to sum up a proper number of radial modes possessing a considerable thermal amplitude; the maximum number is set by the resolution of the instrument - the summation should be stopped at the latest when the first zero of the radial functions becomes comparable to the resolution. As in a real situation $1 / k$ is large compared to the resolution, the series is always truncated earlier by the diminishing thermal amplitude and hence there is no cutoff ambiguity. The two characteristic regimes are illustrated in Fig. 3: a), if $\pi / k \ll R$, many radial modes have to be summed up and the fluctuation amplitude is essentially independent of the system size $R$, whereas b), for $\pi / k \geq R$ (severe confinement) a few modes suffice and the fluctuation amplitude is suppressed by decreasing $R$.

Of course, there exist other soft fluctuation modes, i.e., those with $m \neq 1$, which have not been focused on. Their radial eigenfunctions have zeros at $r=0$, and hence, these modes are not important for the disclination line fluctuations, neither are they particularly characteristic for the defect structure. The $m=0$ soft mode, which involves only the component $x_{-1}$ (Eq. (10) is decoupled in this case), is a special case, corresponding to the Goldstone rotation of $\mathrm{Q}$ around the $z$ axis. Naturally, it is present regardless of the configuration; the defect structure brings about merely a decay of the eigenfunction at the uniaxial core.

Our results are valid in the approximation where the free energy is invariant to separate rotations of space and the order parameter (nematics and smectics- $\mathrm{C}$ in the one constant approximation, superfluids). More precisely, we require that the order parameter gradients in the $z$ direction enter the free energy in the form of square terms only, Eq.(4). If 
this symmetry is broken, the gradients along $z$ are in general coupled to those in the $x y$ plane. In a related manner, superconductors and their analogues, smectics-A (SmA) [20], besides the $X Y$-model (nematics, superfluids) degrees of freedom $\Psi=|\Psi| \exp i \phi$ (wave function of the superconducting state or the amplitude and phase of the smectic modulation) possess an additional vector order parameter (vector potential $\mathbf{A}$ or the c-director, respectively). Due to the coupling between $\nabla \Psi$ and $\mathbf{A}$ described by the free energy density term $|(-i \hbar \nabla-e \mathbf{A}) \Psi|^{2} / 2 \mathrm{~m}$, gradients in the $z$ direction induce a change in $\mathbf{A}$ (analogously for $\mathrm{SmA}$ ). As a consequence, in the string fluctuations the cross-sectional structure of the line defect (vortex or dislocation) is not merely displaced but also deformed, and, hence, the actual line tension is not simply the free energy per unit length (not even asymptotically). In this case one can define an effective line tension and must still renormalize the string model with the contribution of higher radial modes.

This work was supported by the Slovenian Office of Science (Program P1-0099) and US-Slovene NSF Joint Found (Grant No. 9815313).

[1] I. Chuang, N. Turok, and B. Yurke, Phys. Rev. Lett. 66, 2472 (1991).

[2] T. Ishikawa and O. D. Lavrentovich, Europhys. Lett. 41, 171 (1998).

[3] W. Wang, T. Shiwaku, and T. Hashimoto, J. Chem. Phys. 108, 1618 (1998).

[4] S. Thiberge, C. Chevallard, J. M. Gilli, and A. Buka, Liq. Cryst. 26, 1225 (1999).

[5] I. Chuang, R. Durrer, N. Turok, and B. Yurke, Science 251, 1336 (1991).

[6] M. J. Bowick, L. Chandar, E. A. Schiff, and A. M. Srivastava, Science 263, 943 (1994).

[7] T. W. B. Kibble, Physica C 369, 87 (2002).

[8] P. E. Cladis, W. van Saarloos, P. L. Finn, and A. R. Kortan, Phys. Rev. Lett. 58, 222 (1986).

[9] A. Bogi, P. Martinot-Lagarde, I. Dozov, and M. Nobili, Phys. Rev. Lett. 89, 225501 (2002).

[10] A. Mertelj and M. Čopič, submitted.

[11] A. Mertelj, M. Vilfan, and M. Čopič, Mol. Cryst. Liq. Cryst. 395, 311 (2003).

[12] P. G. de Gennes and J. Prost, The Physics of Liquid Crystals (Clarendon Press, Oxford, 1995).

[13] P. Ziherl and S. Žumer, Liq. Cryst. 21, 871 (1996).

[14] P. Ziherl and S. Žumer, Phys. Rev. E 54, 1592 (1996).

[15] E. B. Sonin, J. Low Temp. Phys. 97, 145 (1994).

[16] J. C. Geminard, R. Holyst, and P. Oswald, Phys. Rev. Lett. 78, 1924 (1997).

[17] S. Colson, M. Konczykowski, M. B. Gaifullin, Y. Matsuda, P. Gierlowski, M. Li, P. H. Kes, and C. J. van der Beek, Phys. Rev. Lett. 90, 137002 (2003).

[18] S. Hess, Z. Naturforsch. Teil A 30, 728 (1975).

[19] W. H. Press, B. P. Flannery, S. A. Teukolsky, and W. T. Vetterling, Numerical Recipes (Cambridge University Press, Cambridge, 1986).

[20] P. G. de Gennes, Solid State Commun. 10, 753 (1972). 